\title{
Does the Manchester triage system detect the critically ill?
}

\author{
Matthew W Cooke, Sarah Jinks
}

\begin{abstract}
Background-The Manchester triage system (MTS) is now widely used in UK accident and emergency (A\&E) departments. No clinical outcome studies have yet been published to validate the system. Safety of triage systems is related to the ability to detect the critically ill, which has to be balanced with resource implications of overtriage.
\end{abstract}

Objectives-To determine whether the MTS can reliably detect those subsequently needing admission to critical care areas.

Methods-Analysis of emergency admissions to critical care areas and comparison with original $A \& E$ triage code by a nurse using the MTS at time of presentation. Retrospective coding of all cases according to the MTS by experts and case analysis to determine whether any nonurgent coding was due to the system or to incorrect coding.

Results-Sixty one $(67 \%)$ of the patients admitted to a critical care area were given triage category 1 or 2 (that is, to be seen within 10 minutes of arrival). Eighteen cases given lower priority were due to incorrect coding by the triage nurse. Six cases were correctly coded by the MTS, of which five deteriorated after arrival in the A\&E department. Only one case was critically ill on arrival and yet was coded as able to wait for up to one hour.

Conclusions-The MTS is a sensitive tool for detecting those who subsequently need critical care and are ill on arrival in the A\&E department. It did fail to detect some whom deteriorated after arrival in A\&E. Most errors were due to training problems rather than the system of triage. Analysis of critically ill patients allows easy audit of sensitivity of the MTS but cannot be used to calculate specificity.

( 7 Accid Emerg Med 1999;16:179-181)

Keywords: Manchester triage system; triage; prioritisation; initial assessment

Triage is now universally utilised in accident and emergency (A\&E) departments in the UK. In the past, no benefit has been demonstrated from nurse triage in the $\mathrm{A} \& \mathrm{E}$ department. ${ }^{1}$ It was shown that there was no standardisation of the process or duration of triage. ${ }^{2}$ The Manchester triage system (MTS) was introduced in $1996 .^{3}$ It uses a series of flow charts for various "presentations" with key "discriminators" to determine the triage category. A
Table 1 Cases by triage category

\begin{tabular}{llcc}
\hline Triage category & & $\begin{array}{c}\text { Target time to } \\
\text { be seen (min) }\end{array}$ & Total \\
\hline 1 & & 0 & 21 \\
2 & Immediate & 10 & 39 \\
3 & Very urgent & 60 & 26 \\
4 & Urgent & 120 & 2 \\
5 & Standard & 240 & 0 \\
Not classified & Non-urgent & & 3 \\
Grand total & & & 91 \\
\hline
\end{tabular}

multidisciplinary consensus group developed these guidelines. The system has been adopted widely throughout the UK. There have been no studies published as yet to verify that the consensus group's opinion correlates with the urgency of required clinical care (search of MEDLINE, CINAHL and key journals, plus contact with the Manchester group).

Those patients admitted to critical care areas are by definition among the most seriously ill from the $A \& E$ department. It would be expected that such patients should be triaged to receive care within 10 minutes of arrival in the department and as such should be triaged to category 1 or 2 (definitions in table 1 ). Analysis of these patients provides information on one of the main aims of triage - to select out those requiring immediate attention.

This study aims to test the null hypothesis that the MTS cannot reliably detect those needing subsequent admission to a critical care area.

\section{Methods}

Computerised hospital records at City Hospital, Birmingham were analysed to retrieve details of all patients admitted from the $A \& E$ department to a critical care area (intensive care, high dependency, or coronary care) in a one month period from 10 March 1998 to 9 April 1998. The admission books for these areas were checked to verify that all cases had been included. The computerised $A \& E$ triage notes were retrieved and the triage category of all the critical care area admissions obtained. In those cases where the triage category was not 1 or 2 , the $A \& E$ notes and hospital notes were obtained. Using the information in the notes that could have been available to the triage nurse (initial history and observations) these patients were retrospectively coded according to the MTS guidelines. This includes history obtained later that the triage nurse may have omitted to ask and simple observations that could have been undertaken at triage. This coding was undertaken by a nurse trained in the MTS. The notes were then reviewed to determine why the patient had not been 
Table 2 Cases where the Manchester triage system (MTS) did not code as category 1 or 2

\begin{tabular}{|c|c|c|c|c|c|c|}
\hline $\begin{array}{l}\text { Nurse's } \\
\text { triage } \\
\text { category }\end{array}$ & $\begin{array}{l}\text { Retrospective } \\
\text { triage (MTS) }\end{array}$ & Diagnosis & $\begin{array}{l}\text { Length of stay } \\
\text { in critical care } \\
\text { area (days) }\end{array}$ & Presentation & Discriminator & Reason for apparent undertriage \\
\hline 3 & 3 & Myocardial infarction & 3 & Abdominal pain & Moderate & Presenting with moderate epigastric pain \\
\hline 3 & 5 & SVT & 2 & Mental illness & Marked distress & SVT, not shocked initially \\
\hline 3 & 4 & Asthma & 5 & $\begin{array}{l}\text { Shortness of breath, } \\
\text { adult }\end{array}$ & Wheeze & $\begin{array}{l}\text { Deterioration after arrival in } \mathrm{A} \& \mathrm{E} \\
\text { department }\end{array}$ \\
\hline 3 & 3 & Pneumonia & Died & Collapse & $\begin{array}{l}\text { History of } \\
\text { unconsciousness }\end{array}$ & Sepsis but well compensated initially \\
\hline 3 & 3 & $\begin{array}{l}\text { Guillain-Barré } \\
\text { syndrome }\end{array}$ & 9 & Collapse & Focal loss of function & $\begin{array}{l}\text { Deterioration after arrival in } \mathrm{A} \& \mathrm{E} \\
\text { department }\end{array}$ \\
\hline 3 & 3 & Stroke & Died & Collapse & Focal loss of function & $\begin{array}{l}\text { Deterioration after arrival in } \mathrm{A} \& \mathrm{E} \\
\text { department }\end{array}$ \\
\hline
\end{tabular}

SVT = supraventricular tachycardia.

triaged to category 1 or 2 on arrival in the $A \& E$ department.

\section{Results}

Ninety one patients were admitted to critical areas from the $A \& E$ department during the study period. Seventy one were admitted to coronary care, five to high dependency, and 15 to intensive care. The triage categories of these patients are demonstrated in table 1 . Sixty one patients $(67 \%)$ were appropriately triaged to category 1 or 2 .

In six cases $(6.6 \%)$ of those originally coded as category 3-5, retrospective coding using $A \& E$ and initial history in the admission notes, still gave a triage category of 3,4 , or 5 . These cases are summarised in table 2 . In five of these, initial triage may have given category 3 or 4 correctly as there was an apparent deterioration after arrival. In one case a myocardial infarction presented as moderate epigastric pain with no cardiac symptoms.

In 18 cases that were coded 3-5, retrospective triage coding using MTS guidelines gave a category 1 or 2 . Twelve of these 18 cases were of identifiable cardiac pain $(M T S=2)$, that were classified as 3 in 10 cases and not classified in two cases. One further case of chest pain was associated with sudden onset of

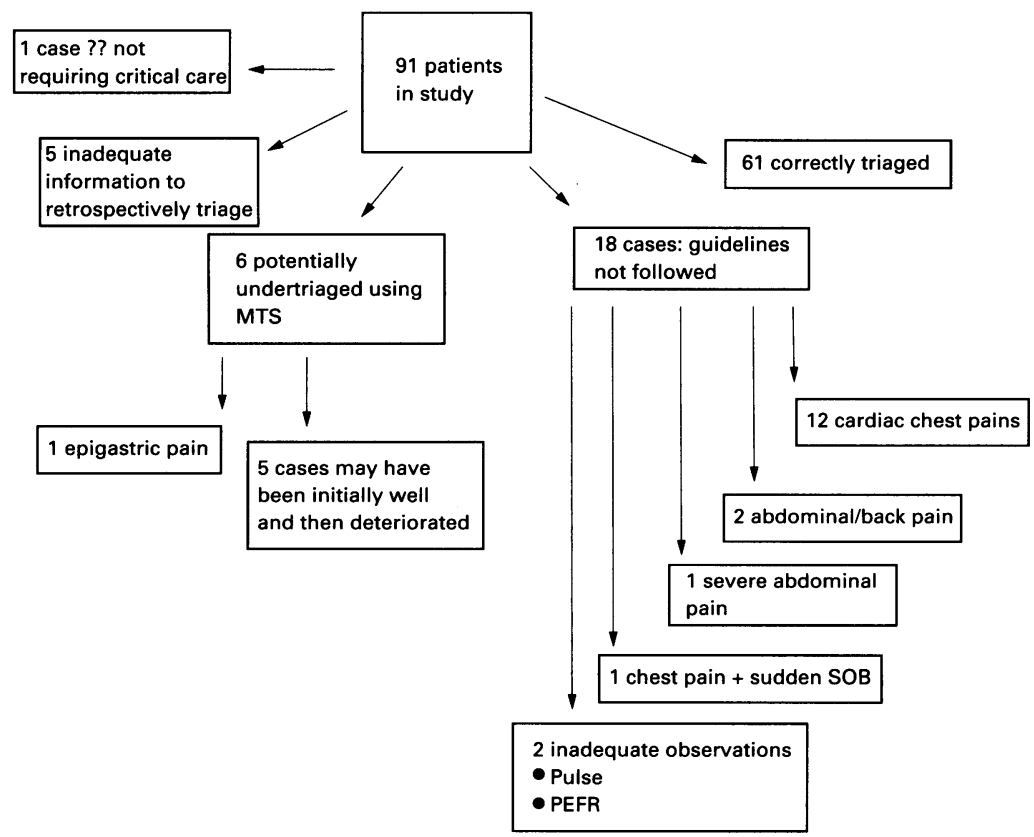

Figure 1 Summary of results $(P E F R=$ peak expiratory flow rates; $S O B=$ shortness of breath). shortness of breath (MTS $=2$ ). One patient with an aneurysm had back pain going to the abdomen and a patient with pancreatitis had abdominal pain radiating to the back. Both were triaged as category $3(\mathrm{MTS}=2)$. One asthmatic had no observations undertaken and therefore his peak expiratory flow rate of $160 \mathrm{l} / \mathrm{min}$ and pulse of 120 beats/min were not noted and he was triaged as category 3 . There was one case each of severe abdominal pain and collapse with a tachycardia. The results are summarised in fig 1 .

Of those cases where the triage nurse underscored compared with the MTS, 12 were self referrals or 999 calls and six $(33.0 \%)$ were general practitioner (GP) referrals. This compares with $17.6 \%$ (16 of 91 ) of the whole study population. Analysis revealed that these 18 cases were triaged by 16 different nurses.

In five cases it was not possible retrospectively to triage the patient because of lost notes or inadequate recorded information. Two of these cases simply stated "see GP letter" with no evidence of assessment at the triage area. One further case had no apparent reason for admission to a critical care area, even on final discharge.

\section{Limitations of study}

We have no reason to believe that the one month period selected is atypical or that the hospital investigated should produce figures different from other inner city $A \& E$ departments. Checking of manual and computer records did not reveal any missing cases. This is the first study of its kind and therefore numbers are small and further larger studies are required to provide more information. Only the triage nurse can assess the pain of the patient and this cannot be assessed retrospectively. However the patients undertriaged with any pain also had other definable reasons for inclusion in a higher category.

\section{Discussion}

The MTS failed to detect six patients admitted to critical care areas. However in five cases this was because the patient's condition deteriorated after arrival in the $A \& E$ department. This illustrates the importance of triage being a dynamic process. In one case the patient had sustained a myocardial infarction but did not present with typical cardiac pain. The pain was epigastric and of only moderate severity with no other cardiac symptoms or past medical 
history. We do not believe such cases could be detected in a triage system without adding a vast number of patients with abdominal pain to an excessively high category. It is possible that such cases could be triaged to have immediate electrocardiography and monitoring without urgency of medical care.

This study demonstrates that the MTS is a sensitive tool for detecting the critically ill. Only one case was demonstrated where it may have been apparent that the person was seriously ill at triage (five others admitted to critical care areas deteriorated after triage) and not detected by the MTS guidelines. The small numbers in this pilot study will not allow calculation of a significant sensitivity. The specificity of the Manchester system cannot be determined using this methodology. We do not believe that this study has demonstrated any area of the guidelines that need modification.

The majority of the errors in this study were in a failure to correctly apply the MTS. All the staff involved had been trained in the system and had a copy of the manual. Stricter adherence to the MTS may be achieved by using documentation that requires the "presentation" and "discriminator" to be recorded in all cases. The commonest mistriaged group was those with chest pain. Eight of the 12 cases of chest pain who were mistriaged gave a clear past medical history of cardiac disease and continuing chest pain. The others all had clear cardiac features. More work is needed to determine why the triage nurses failed to give these cases appropriate urgency. This group is vitally important as it is known that the earlier thrombolysis is started the more effective it is ${ }^{4}$ and yet only about $5 \%$ of patients presenting with chest pains to A\&E departments have a myocardial infarction. ${ }^{5}$ Three patients would have been detected if appropriate observations had been undertaken. Pulse oximetry has been demonstrated to be a useful triage tool, resulting in changes in management. ${ }^{6}$ Those with back and abdominal pain were missed because of failure to detect the referred pain as a guide to diagnosis. The study has highlighted key areas for further education in relation to triage.

The higher percentage of GP cases in the triage nurse error group may represent poor quality of triage for patients referred directly to specialties by the GP. After discussion with the staff involved, it is possible that this is because the triage nurses perceive that specialist teams take little notice of the triage category, compared with A\&E medical staff. As GP referrals often have a delay in assessment, it is therefore important that triage is undertaken on these cases to ensure the critically ill receive care without delay.

It is difficult to monitor triage with clinical outcome because of the large numbers involved. Expert opinion from retrospective review has demonstrated a high rate of disagreement about urgency even when working to the same criteria. ${ }^{7}$ Video review of triage has also been used. ${ }^{8}$ Random card audits may be helpful. Analysis of 10000 cases in the same time period as this study revealed that $50.3 \%$ of cases were category 4 and $34.2 \%$ were category 3. Random card audits may therefore fail to highlight triage problems because of the dilution of errors by the vast majority of cases being in categories 3 or 4 . A prime aim of triage is to select out those who cannot afford to wait. Hence, we believe that analysis of critical care admissions provides an effective focused method of triage audit without an excessive workload. Subsequent training can then be directed to the areas where triage errors are occurring.

We would like to thank Sue Smith and Sue Wilson for their helpful comments on the early drafts of this paper.

Conflict of interest: none.

Funding: SJ was funded by the Pulse Trust during the study.

1 George S, Read S, Westlake L, et al. Evaluation of nurse triage in a British accident and emergency department. BMF 1992;304:876-8

2 Edhouse JA, Wardrope J. Do the national performance tables really indicate the performance of accident and emergency departments. $\mathcal{F}$ Accid Emerg Med 1996;13:1236.

3 Manchester Triage Group. Mackway-Jones K, ed. Emergency triage. London: BMJ Publishing Group, 1997.

4 Weaver WD. Time to thrombolytic treatment: factors affecting delay and their influence on outcome. $\mathcal{F} \mathrm{Am}$ Coll Cardiol 1995;25(suppl):3S-9.

5 Ornato JP. Problems faced by the urban emergency department in providing rapid triage and intervention for the patient with suspected acute myocardial infarction. Heart pung 1991;20:584-8.

6 Mower WR, Sachs C, Nicklin EL, et al. Effect of routine emergency department triage pulse oximetry screening on emergency department triage pulse oximetry scre

7 Gill JM, Reese CL, Diamond JJ. Disagreement among health-care professionals about the urgent care needs of emergency department patients. Ann Emerg Med 1996;28 474-9.

8 Williams JC, Jones NL, Richardson FJ, et al. The nursing triage process-a video review and a proposed audit tool. $f$ Accid Emerg Med 1996;13:398-9. 